\title{
REFLEXIÓN
}

\section{ENTRE LA TECNOLOGÍA DE LA INFORMACIÓN Y LA PEDAGOGÍA}

JORGE E. BENAVIDES B.

Universidad de Nariño, RUDECOLOMBIA, Colombia

\section{INTRODUCCIÓN}

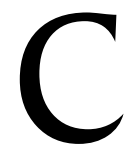
ada día el curso de las cosas parece hacerse más veloz, los cambios son más rápidos en la gran mayoría de los ámbitos del quehacer humanos y esta aceleración de la historia que estamos viviendo, síntoma, causa y efecto de la globalización, en todas sus vertientes, y del desarrollo de las tecnologías de la información, característica de los periodos de transición está provocando que la sociedad demande crecientemente que la historia se reescriba. Así el papel que juega la educación en un contexto global crece en relación con las tendencias nacionales e internacionales, gracias a las nuevas tecnologías de la información y la comunicación y a los procesos de integración transnacional en el terreno económico, político, cultural y académico.
En el siglo XXI la educación y la pedagogía parecen tener más que ver con las nuevas tecnologías de la información y la comunicación de cada país y están, más que nunca condicionadas por esas tendencias internacionales. Es importante pues hacer una reflexión sobre la relación entre las nuevas tecnologías de la información y la pedagogía, su campo de acción, lo que se esta haciendo y lo que tal vez se está dejando de hacer.

\section{Aproximación a la pedagogía de la tecnología de la información}

Al parecer el campo de la tecnología es considerado más como una forma de pensar que va de la mano con la cultura actual y con la ayuda de la velocidad de desarrollo de la ciencia computacional, la proliferación de dispositivos informáticos y la acción de los medios masivos de comunicación. Este contexto ha permitido al ser hu- 
mano llegar a "imaginarse lo inimaginado"1 o elaborar todo un mundo de posibilidades de enseñanza a través del uso de las tecnologías que se tiene a la mano, pero sin las necesarias adecuaciones o adaptaciones al entorno. Aplicar el mundo en que se vive a la vida escolar sería un hecho de por si encomiable, sin embargo esto no ha pasado del sueño o de algunas realizaciones sin un método explícito.

Tal vez quien mejor se acerca al carácter práctico de la pedagogía utilizando lo que se tiene a la mano en la vida diaria sea Celestin Freinet, quien aproxima de manera pedagógica la vida de la cultura del ser a la práctica de la vida, es decir, el método que permitiese al ser humano aprender en la escuela como aprende en la vida, algo que generalmente es ignorado por el anhelo de formalización y estandarización de lo real.

La filosofía de la educación suele confundirse con las técnicas, en este caso en particular con la tecnología de la información confundiendo los fines educativos con los fines que persiguen la tecnologías. Sin embargo, esto no quiere decir que la educación tenga que oponerse a las tecnologías, por el contrario, es, ante todo, una posibilidad de entender la educación y el proceso educativo haciendo uso de la tecnología de la información, producto del desarrollo tecnológico de nuestro tiempo.

La actividad diaria del ser humano está incondicionalmente relacionada con el trabajo, y su noción no ha sido desarrollada como una noción de aproximación a la realidad y como mecanismo de práctica educativa, siendo este concepto el que los niños, y los jóvenes tienen que afrontar después de su vida escolar. El trabajo, según Freinet, es una de las actividades más or ganizadas de la vida diaria y que extiende sus posibilidades pedagógica al carácter de cooperación, orden, responsabilidad, valor, etc. y que muestra como meta un accionar social y cultural de innegable fuerza y cognición humanas.

La pedagogía frente al reto de la tecnolo-gía como estructura del mundo, según Var-gas (1999) es una realidad que tal vez no hemos podido entender en primera instancia ni asumir dentro de nuestro papel de educadores. De esta manera no se ha permi-tido explotar las posibilidades pedagógicas de la tecnología, ya sea por temor natural a lo nuevo, por la inseguridad ante lo desconocido, el desinterés injustificado o por la prevención tecnofóbica del educador frente a la posibilidad del desplazamiento del educador en el trabajo y mayormente por la falta de capacitación. La mirada a la tecnología de la información como un componente de todo educador como posibilidad pedagógica no intenta sino ser un "rodeo o -estratagema- tomado de la cultura actual que procura el reconocimiento de los nuevos escenarios que se abren para la formación (subjetiva-intersubjetiva) y para la enseñanza; es volver la mirada sobre el ho-rizonte de realización que compete asumir a quienes se forman y se encuentran en pro-ceso de comunicación tanto de idealidades como de estructuras simbólicas" (p. 120).

\section{La tecnología de la información como experiencia del mundo}

En educación sucede algo completamente diferente con la introducción de las tecnologías de la información. Es algo que está (o ha creado) creando angustia o ansiedad de estar relegado al no uso de la tecnología existente para tareas de enseñan-

1. A la manera de la tradición de la filosofía del Heidegger como una forma de "pensar lo impensado". 
za y aprendizaje como es el uso de la tecnología informática. Se está en frente a la contradicción de que siendo la tecnología de la información parte de una experiencia del mundo de la vida que se utiliza cada vez más por nosotros mismos no le hemos considerado dentro de una concepción educativa. Incluso, hasta tal punto como lo manifiesta Vargas (1999) de que se la considera casi como un "objeto de bienestar" y cada vez son más comunes las expresiones de descontento de la gente (maestros, educadores, incluidos) cuando se quejan de que "todavía no tienen computadores" "no están sistematizados!" "Se demora, porque no tenemos computador", etc.

Muchas son las situaciones en que el profesor para no quedarse atrás de la "etiqueta"informática, pide a sus estudiantes realizar determinado trabajo haciendo uso del computador o de investigar en Internet, frente a la posibilidad de no acceso a fuentes de consulta. Sin embargo, lo más probable es que el profesor no haya realizado la práctica auténtica, -en este caso de nivel investigativo, que él mismo demanda a sus estudiantes: Un caso de práctica formal y experiencia pedagógica. A nivel universitario ya prácticamente no existe la excusa "no tengo computador"o "no tenemos Internet", se cuenta con este servicio -así no sea el mejor, pero lo crítico de la situación pedagógica de la tecnología de la información es que no se ha realizado suficiente reflexión sobre la misma, ni del tratamiento que esta implica en un proceso de educación y de formación.

El uso de la tecnología parece no necesitar reflexión, sino acción en el mundo de la experiencia de la vida diaria, es decir de aplicación en el trabajo. Es mucho más instrumental la relación con la tecnología toda vez que se enfatiza más sobre el dispositi- vo que sobre el sentido. Sin embargo, el uso de la tecnología de la información en educación, debe ser un campo de reflexión pedagógica.

\section{La formación de pertenencia social de los profesores}

La enseñanza cumple un papel importante dentro de la transmisión de los valores de determinada cultura y sus agentes de transmisión son los mismos profesores quienes se encargan de mantenerla según los mismos intereses y valores que se comparten. Es por esto que los profesores tienen que estar preparados para afrontar el reto que impone el mismo cambio, las innovaciones, los intereses particulares, los del mercado, los de la empresa privada, las tensiones y presiones externas, etc. Son los encargados al mismo tiempo de la formación de qué tan necesario es el cambio, cuales son las ventajas y los peligros del mismo frente a los intereses y valores sociales y culturales. De ahí que es importante la formación del profesorado universitario tanto o más importante que el componente del saber específico o profesional ya que están de por medio las decisiones sobre el mantenimiento de los valores socioculturales dentro del quehacer universitario y social.

Esta formación, a través de los programas específicos de docencia universitaria pueden marcar la diferencia entre la producción del nuevo conocimiento y su pertinencia con la sociedad, la democratización de ese conocimiento en forma de acción como conocimiento práctico y su aplicación en las necesidades de desarrollo de la sociedad. La apropiada comunicación de los nuevos conocimientos desarrollados en el ámbito universitario, la difusión social del mismo y su aplicación son pues los pro- 
cedimientos que de alguna manera asegurarían que el nuevo conocimiento producido en la universidad se revierta en la sociedad como primera meta del mismo en forma de acción social.

Pero qué herramienta o mecanismo podría acercarse para que a lado de la formación del profesorado universitario garantice la apropiada difusión, apropiación y aplicación de ese nuevo conocimiento? Una vez más la tecnología a través de un proceso de formación pedagógica puede dar una posible respuesta a las necesidades de acción social.

Si somos realistas, nuestros esfuerzos por dar prioridad a la metodología, la pedagogía y la epistemología chocan por la falta de formación -cuando no de interés, lo que es peor- de una gran parte de los educadores profesionales ${ }^{2}$, lo cual sumado a la reiterada posición de actitud conformista conduce, en ocasiones, a una minus-valoración curricular de este tipo de trabajos de investigación y reflexión, pese a la progresiva admiración intelectual que provocan entre quienes trabajan a diario con las nuevas tecnologías de la información. Este desfase entre formación y necesidades nos plantea la cuestión de la formación continúa del profesorado universitario, en este caso de historia. La evolución del conocimiento, acelerada en los últimos tiempos, y las nuevas tecnologías, obligan a un reciclaje del profesorado en la enseñanza superior que, mientras no se impulse de manera institucional, hay que suplir con esfuerzos individuales y colectivos que se apoyen en los sectores más dinámicos, menos gre- o conformistas, de las comunidades de educadores.

\section{El aspecto Interdisciplinar de la tecnología en el mundo}

La primera insuficiencia del principio de interdisciplinariedad en el inicio al estudio de la pedagogía desde la introducción en los años 80's es su restricción a determinadas ciencias como las matemáticas, la informática, la ingeniería de sistemas, etc. Este campo sólo se podrá afirmar como en igualdad con otras ciencias sociales y disciplinas humanísticas, si se aplica a si misma el criterio metodológico y epistemológico de la interdisciplinariedad y extiende, al mismo tiempo, el diálogo a todas los saberes, desde las humanidades (filosofía y literatura, sobre todo) hasta las ciencias de la naturaleza ${ }^{3}$, pasando por las nuevas disciplinas surgidas de la sociedad de la información y las nuevas tecnologías.

Una cuestión a decidir es si los nuevos enfoques globales formarán parte de procesos de investigación pedagógica o se reducirán como hasta ahora a modos de exponer sintéticamente, con fines didácticos, los productos de unas investigaciones especializadas, fragmentadas, con un resultado a menudo indigerible para el lector. La racionalidad del campo pedagógico debería animarnos a ensayar aproximaciones globales que unifiquen la investigación y la divulgación. Sería desde luego la mejor opción, y las nuevas tecnologías favorecen enormemente dicha convergencia, pero, como bien sabemos, la racionalidad epistemológica y metodológica no lo es

2. El número de los que optan por la auto-formación no es muy grande. Pero sí de los que toman cursos en tecnología de la información aunque no sea para aspectos que se relacionen con educación o pedagogía.

3. La historia del ser humano no puede ser entendida sin la historia del mundo natural en el que vive y de la cultura, incluida la tecnología de que se apropia. 
todo, y a veces ni siquiera es lo más importante a la hora de las decisiones de una comunidad de especialistas, en este caso, compuesta de educadores.

La globalización y nuevas tecnologías hacen posible una contexto pedagógico más global en el sentido de crear condiciones técnicas para una aproximación más integral a nuestros objetos. Porque los problemas habidos para llevar a la práctica el paradigma renovador de la pedagogía también se deben a los límites físicos del soporte utilizado para plasmar las investigaciones en el campo: el libro con su número limitado de páginas y su modo de lectura lineal, con un único principio y final, y la sola potestad de unas breves citas en el texto o notas a pie de página. Sin embargo, la técnica del CD-ROM posibilita incluir, en la fase de investigación y/o en la fase de divulgación, además de textos escritos, el componente oral y visual del sonido y del vídeo, con lo cual nos acercamos a la percepción global de la realidad que nos permiten cotidianamente los cinco sentidos humanos.

Pero es el hipertexto, que manejamos cotidianamente navegando por la Web, junto con los recursos multimedia, lo que puede transformar el proceso de investigación pedagógica y el proceso de divulgación en un todo, aproximando la práctica tradicionalmente analítica y fragmentadora de las ciencias sociales a una realidad que sabemos esencialmente sintética, compleja y global? Las citas y notas son ahora enlaces que nos trasladan a textos con la longitud que se deseen, dentro de los cuales podemos incluir asimismo otros vínculos que conduzcan a otros textos (sonidos o imágenes). Con lo cual la obra puede tener más de un principio y más de un final, además de una difusión en la red de redes virtualmente mayor que un libro.
Esta nueva técnica de hipertexto y multimedia, llamadas hipermedia, en formato CD-ROM o Internet, se está aplicando ya a la enseñanza y la divulgación de casi todos los saberes. La incorporación de Internet en todos los niveles de la enseñanza beneficiará el cambio de mentalidades indispensable para universalizar a plazo medio el uso de las nuevas tecnologías, también en la investigación pedagógica, cuando sea pertinente, es decir, cuando precisemos aproximaciones cruzando textos, sonidos, imágenes y vídeos, lo cual dependerá de las fuentes disponibles y de nuestra capacidad intelectual para articular y sintetizar datos tan diversos.

\section{Un caso investigativo en educación Básica}

Es muy conocido el nuestro medio latinoamericano la aceptación de la tecnología sin mediar procesos de reflexión, crítica y evaluación y de preocupación institucional que sirva de filtro moderador al muy frecuentemente desbordado entusiasmo por lo nuevo que provenga de afuera. Este es el caso de las nuevas tecnologías de la información y particularmente la tecnología informática representada en el uso del computador en la cotidianidad: trabajo, comercio, administración, educación etc.

En el campo educativo es todavía más interesante el hecho de asumir posturas de implicaciones cognitivas atribuidas al uso y aplicación de la tecnología de la información. La indudable validez física de los aparatos ha determinado su introducción como instrumentos educativos válidos $\mathrm{y}$ provechosos puestos en manos de profesores y estudiantes.

Sin embargo, es poco lo que se sabe a través de la investigación y el conocimien- 
to científico sobre el potencial "cognitivo" como impacto real de la T.I. en educación y sobretodo su aproximación pedagógica a nuestra realidad. Estudios diagnósticos recientes (Benavides, 1999, 2000) muestran que contrariamente a la gran aceptación de esta en una etapa de introducción se ha puesto de manifiesto los siguientes aspectos desde la perspectiva de los estudiantes, profesores y espacio físico (no previstos por el sistema):

\section{Las instituciones y los profesores}

- Es evidente la necesidad de la alfabetización informática y de capacitación pedagógica para los profesores.

- Hay necesidad de tener claridad sobre la integración del componente de la tecnología de la información al currículo, en aspectos formales como el PEI

- Hay que rescatar la actitud positiva del profesor y de estudiantes frente al uso y aplicación de la tecnología de la información (El buen uso de la tecnología)

- La tecnofobia es un resultado de la falta de capacitación del profesorado, ya que solo se da en este sector.

- Se necesita urgentemente la preparación pedagógica de los profesores para precisamente hacer frente a problemas de aprendizaje que pueden surgir.

- Los profesores tendrían que reevaluar su propio trabajo si su meta educativa está en hacer del aprendizaje algo más dependiente de la interacción entre los aprendices. Esto conduce a pensar que el aprendizaje colaborativo integra el componente social buscado por Freire, y Vygotsky.

\section{Sobre los estudiantes y el nuevo contexto educativo}

- El nuevo contexto, según la posición vygotskiana del aprendizaje, permitiría que tanto profesores como estudiantes trabajen en forma más integrada y cercana en cuanto a intereses y metas (humanismo).

- Promover tanto el Aprendizaje Exploratorio y por descubrimiento involucrando a los profesores en un papel de planificación y creatividad en el desarrollo del aprendizaje de los estudiantes. Es más probable que los estudiantes que trabajan en este tipo de contextos aprendan e internalicen más rápidamente los frutos de sus propios descubrimientos (Stevens, 1992).

- Enfatizar el trabajo del estudiante en pro del desarrollo del Aprendizaje Colaborativo. Según el punto de vista vygotskiano del aprendizaje el papel del profesor es el de un ayudante donde su papel comunicativo con los aprendices tiende a ser el centro de toda actividad.

- Se destaca el papel central de los materiales y las tareas desarrolladas cuando existe compromiso de los aprendices en la tarea. Compromiso con la tarea aprovechando la alta validez física del computador y el software. Podría ser el hecho de tener que hablar acerca de lo que se está haciendo al realizar una tarea o resolver un problema lo que hace que el aprendizaje sea más efectivo Light (1993).

- Este nuevo contexto presenta un ambiente menos intimidante para el estudiante donde este aprende a ser autónomo y se sumerge en un proceso educativo ideal, como en el caso de la clínica (Rogers, 1969). 
- Este nuevo contexto amerita un tratamiento especial basado en la investigación, observación y compromiso de todo el sistema educativo. Entender mejor los procesos de aprendizaje y de desarrollo a través de la investigación.

- El estudiante debe ser tratado como un sujeto que está en un contexto diferente que amerita nuevas características, y por consiguiente se encuentra frente a la posibilidad de desarrollar nuevas estrategias de aprendizaje y nuevos comportamientos. No se puede desconocer el hecho de que siempre que se realiza algún nuevo tipo de actividad se asume que se está frente al desarrollo de un tipo nuevo de estrategia cognitiva para hacerlo.

\section{Sobre la infraestructura (locatividad)}

- La adecuación física de las aulas de computadores deben ofrecer un mínimo de comodidad y espacio para que los estudiantes puedan realizar adecuadamente su trabajo.

- Explorar formas de financiación de la infraestructura en el sentido de proveer con mayor número de computadores para que al menos (pedagógicamente hablando) pueda haber un computador por cada dos estudiantes. Sin embargo es preciso que no se descuiden los aspectos de diseño, espacio, y comodidad.

- La inversión en infraestructura debe estar equiparada en la misma proporción con la que se debe hacer en capacitación del personal docente.

- No se necesita de un sofisticado equipo para logra iniciar la aplicación de este nuevo contexto, sin embargo es definitivo la disposición y actitud de un conglomerado de sujetos que tienen que ver con este aspecto para mejorarlo y propender por su desarrollo pedagógico con el apoyo a la investigación para poder tener a esta última la base del desarrollo de un modelo pedagógico y metodológico propio en la aplicación de la tecnología de la información.

Se puede notar la urgente necesidad de un plan de capacitación de investigación y seguimiento de la aplicación de la tecnología de la información para poder iniciar el proceso de desarrollo pedagógico y del uso adecuado de la tecnología para que esté al servicio del hombre y de la mejoría de la calidad de la educación.

\section{A manera de Conclusión}

La conclusión de esta charla es practicamente un llamado a la comunidad de educadores, no sólo del campo universitario y de los profesores o estudiantes sino de la comunidad en general en el sentido de considerar en su reflexión educativa y formativa de las nuevas generaciones. La toma de las mejores decisiones está mediada por la reflexión, la investigación y la comunicación. Este proceso deber considerarse seriamente en un mundo donde la tecnificación ha tomado fuerza sin cuestio-namientos.

En realidad, no sabemos en que medida se están dando estos cambios y en qué campos de la educación superior son más evidentes. La duda real sería que la velocidad y la evidente inevitabilidad de la aplicación de la tecnología persuada a los profesores que ellos no tienen que ver más en la toma de decisiones sobre el futuro de la educación. Puede discutirse con argumentos de bastante fuerza, que los profesores deben continuar viendo su papel como el del que forma, dirige, y orienta con sus 
estudiantes tanto la marcha como la dirección de la innovación: la introducción del computador e Internet en el aula y fuera ella como un sistema de libre acceso tiene que acercar a la comunidad educativa e investigativa a considerar estos cambios y la pertinencia de los mismos. Esto, por su puesto involucra mucha reflexión y evaluación sistemática. Los profesores debemos estar abiertos a las innovaciones, como con el uso cotidiano de Internet en educación superior, pero alertas y al mismo tiempo constructivamente críticos de las mismas.

\section{BIBLIOGRAFÍA}

BENAVIDES, J. (1999, 2000). Estudio diagnóstico en Informática Educativa en Educación básica para capacitación y desarrollo pedagógico. San Juan de Pasto, Universidad de Nariño (trabajo de investigación, sin publicar).

FREINET, Celestin. (1979) Los métodos naturales. Barcelona: Fontanella.

LIGHT, P. (1993). 'Collaborative Learning with Computers'. In P. Crimshaw. (ed.) Language classrooms and computers. London: Routledge.

LIGHT, P.H. \& GLACHAN, M. (1985). 'Facilitation of individual problem solving through peer interaction', Educational Psychology, 5: 217-25.

ROGERS, C. (1961). On Becoming a Person: A therapist's View of Psychotherapy. Lonson: Constable.

ROGERS, C. (1969). Freedom to learn. Colombus, OH: Chas. E. Merrill Publishing Co.

STEVENS, V. (1992). 'Humanism and CALL: A coming of Age'. In M. Pennington, \& V. Stevens (eds.). Computers in Applied Linguistics. Clevedon: Multilingual Matters Ltd.

VARGAS, G. Germán. (1999). Filosofía, pedagogía, tecnología, Santafé de Bogotá: Universidad de San Buenaventura. 